\title{
Secret Key Capacity For Multipleaccess Channel With Public Feedback
}

\author{
Himanshu Tyagi*
}

\author{
Shun Watanabe ${ }^{\dagger}$
}

\begin{abstract}
We consider the generation of a secret key (SK) by the inputs and the output of a secure multipleaccess channel (MAC) that additionally have access to a noiseless public communication channel. Under specific restrictions on the protocols, we derive various upper bounds on the rate of such SKs. Specifically, if the public communication consists of only the feedback from the output terminal, then the rate of SKs that can be generated is bounded above by the maximum symmetric rate $R_{f}^{*}$ in the capacity region of the MAC with feedback. On the other hand, if the public communication is allowed only before and after the transmission over the MAC, then the rate of SKs is bounded above by the maximum symmetric rate $R^{*}$ in the capacity region of the MAC without feedback. Furthermore, for a symmetric MAC, we present a scheme that generates an SK of rate $R_{f}^{*}$, improving the best previously known achievable rate $R^{*}$. An application of our results establishes the SK capacity for adder MAC, without any restriction on the protocols.
\end{abstract}

\section{INTRODUCTION}

What is the largest rate of a secret key (SK) that can be generated by the inputs and the output of a secure multipleaccess channel (MAC) with a public feedback from the output? We show that this rate is bounded above by

$$
R_{f}^{*}=\max \left\{R:(R, R) \in \mathcal{C}_{\mathrm{MACFB}}\right\},
$$

where $\mathcal{C}_{\text {MACFB }}$ denotes the capacity region 1$]$ of the MAC with feedback. In fact, for a MAC that is symmetric with respect to its inputs, this largest SK rate is equal to $R_{f}^{*}$.

Previously, Csiszár and Narayan [6] presented two different protocols to establish SKs of rate

$$
R^{*}=\max \left\{R:(R, R) \in \mathcal{C}_{\mathrm{MAC}}\right\},
$$

where $\mathcal{C}_{\text {MAC }}$ denotes the capacity region of the MAC without feedback. In both the protocols, the inputs of the MAC were selected without any knowledge of the previous outputs. Such protocols are reminiscent of SK generation in source models [4] and will be collectively referred to as source

\footnotetext{
*Department of Electrical and Computer Engineering, and Institute for Systems Research, University of Maryland, College Park, MD 20742, USA. Email: tyagi@umd.edu

${ }^{\dagger}$ Department of Information Science and Intelligent Systems, University of Tokushima, Tokushima 770-8506, Japan, and Institute for Systems Research, University of Maryland, College Park, MD 20742, USA. Email: shunwata@is.tokushima-u.ac.jp

Himanshu Tyagi was supported by the U.S. National Science Foundation under Grants CCF0830697 and CCF1117546.

${ }^{1}$ Throughout this paper, the capacity region of the MAC is for the average probability of error criterion.
}

emulation 2. We show that $R^{*}$ is the best rate of an SK that can be generated using such simple protocols. Since for symmetric MACs we generate an SK of rate $R_{f}^{*}$, it follows that complex protocols that select inputs of the MAC based on the feedback from the output can outperform source emulation. This answers a question raised in [6, Section VII].

In general, the inputs of the MAC can be selected based on interactive public communication from all the terminals after each transmission over the secure MAC. For this setup, Csiszár and Narayan established an upper bound for the largest rate of an SK [6], termed the SK capacity and denoted by $C$. Moreover, for the special case of MACs in Willems class [12], this upper bound was improved and it was shown that $C \leq R_{f}^{*}$. Therefore, for symmetric MACs in Willems class, our aforementioned results imply $C=R_{f}^{*}$. This class of channels includes adder MAC, which settles an open problem posed in [6, Example 2].

One of the rate $R^{*}$-achieving schemes in [6] involves transmitting messages $M_{1}, M_{2}$ of rates $\left(R^{*}, R^{*}\right)$ over the MAC and communicating the modulo sum $M_{1} \oplus M_{2}$ over the public channel, resulting in an SK of rate $R^{*}$; either $M_{1}$ or $M_{2}$ constitutes the SK. It was remarked in 6 page 21] that an SK generation protocol with "full feedback is ruled out as the feedback communication is public. Still, if a coding scheme with partial feedback could be found by which the gain in transmission rates exceeds the information leakage due to feedback, it would lead to an SK rate greater than" $R^{*}$. Following this clue, our achievability scheme for symmetric MACs entails communicating compressed output sequences over the public channel and then extracting an SK of rate $R_{f}^{*}$ from the output sequence. One difficulty is the lack of a single-letter expression for $R_{f}^{*}$. However, this is circumvented by converting the transmission schemes for MAC directly into SK generation protocols, without recourse to the single-letter rate achieved. In fact, our approach implies that any message transmission scheme of rates $(R, R)$ for a symmetric MAC can be used to generate an SK of rate $R$, with appropriate modifications.

Our converse proofs rely on a general converse 3 for the SK generation problem in a multiterminal source model, which in turn is a simple consequence of a basic property of interactive

\footnotetext{
${ }^{2}$ Our source emulation protocols include the generalized source emulation of [6], [3] as a special case; the latter restricts the MAC inputs for different channel uses to be independent and identically distributed (i.i.d.).

${ }^{3}$ This general converse is due to Prakash Narayan, who agreed to publish it in this paper.
} 
communication that was established in [5, Lemma B.1] (see, also, [8]). Here, too, the challenge posed by the lack of singleletter expressions is handled by working directly with $n$-letter expressions.

The problem formulation and our main results are stated formally in the following section. Sections $\Pi$ and IV contain the necessary tools that are used in our converse proofs in Section $\mathrm{V}$. The final section contains a discussion of our results and the properties of interactive communication that are used to derive them.

\section{Problem Formulation And Main Results}

Consider a MAC with two inputs $\mathcal{X}_{1}$ and $\mathcal{X}_{2}$, and an output $\mathcal{X}_{3}$, specified by a DMC $W: \mathcal{X}_{1} \times \mathcal{X}_{2} \rightarrow \mathcal{X}_{3}$. We study a secrecy generation problem for three terminals: terminals 1 and 2 govern the inputs to the DMC over which they transmit, respectively, sequences $\mathbf{x}_{1}$ and $\mathbf{x}_{2}$ of length $n$, while terminal 3 observes the corresponding $n$ length output $\mathbf{x}_{3}$. Between two consecutive transmissions, the terminals communicate with each other interactively over a noiseless public communication channel of unlimited capacity. While the transmissions over the DMC $W$ are secure, the public communication is observed by all the terminals as well as a (passive) eavesdropper. This model is a special case of a general model for secrecy generation over channels introduced by Csiszár and Narayan in [6] (see also [5]). In the manner of [6], the messages sent over $W$ will be referred to as transmissions and those sent over the public channel will be referred to as communication.

Formally, assume that at the outset terminal $i$ generates rv $U_{i}, i=1,2,3$, to be used for (local) randomization; the rvs $U_{1}, U_{2}, U_{3}$ are mutually independent. The communicationtransmission protocol can be divided into $n+1$ time slots. In the first $n$ time slots, the terminals communicate interactively over the public channel, followed by a transmission over the secure DMC. The protocol ends with a final round of interactive public communication in slot $n+1$. Specifically, in time slot $t, 1 \leq t \leq n$, the terminals communicate interactively using their respective local randomization $U_{1}, U_{2}, U_{3}$ and observations upto time slot $t-1$; the overall interactive communication in slot $t$ is denoted by

$$
F_{t}=F_{t}\left(U_{1}, U_{2}, U_{3}, X_{3}^{t-1}, F^{t-1}\right)
$$

Subsequently, the inputs $X_{1 t}=X_{1 t}\left(F^{t}, U_{1}\right)$ and $X_{2 t}=$ $X_{2 t}\left(F^{t}, U_{2}\right)$ are transmitted by terminals 1 and 2 , respectively, and $X_{3 t}$ is observed by terminal 3. Finally, the last round of interactive communication $F_{n+1}=$ $F_{n+1}\left(U_{1}, U_{2}, U_{3}, X_{3}^{n}, F^{n}\right)$ is sent over the public channel. For convenience, we denote $\mathbf{F}=\left(F_{1}, \ldots, F_{n+1}\right)$.

After the communication-transmission protocol ends, the terminals $1,2,3$, respectively, form estimates $K_{1}, K_{2}, K_{3}$ as follows:

$$
K_{i}=K_{i}\left(U_{1}, \mathbf{F}\right), \quad i=1,2,3 .
$$

\footnotetext{
${ }^{4}$ Our results in this paper can be extended to the multiple input case. See Section VII
}

An rv $K$ with range $\mathcal{K}$ constitutes an $\epsilon$-SK if the following two conditions are satisfied (c.f. [4]):

$$
\begin{aligned}
& \mathrm{P}\left(K_{1}=K_{2}=K_{3}=K\right) \geq 1-\epsilon, \\
& s_{i n}(K ; \mathbf{F}):=\log |\mathcal{K}|-H(K \mid \mathbf{F}) \\
&=D\left(\mathrm{P}_{K \mathbf{F}} \| \mathrm{P}_{\text {unif }} \times \mathrm{P}_{\mathbf{F}}\right) \\
& \leq \epsilon,
\end{aligned}
$$

where $\mathrm{P}_{\text {unif }}$ is the uniform distribution on $\mathcal{K}$. The first condition above represents reliable recoverability of the SK and the second guarantees its security. While our achievability proofs establish SKs that satisfy the "strong secrecy" condition (6), our converse results are valid for SKs satisfying the weaker secrecy condition given below:

$$
\frac{1}{n} s_{i n}(K ; \mathbf{F}) \leq \epsilon .
$$

Definition 1. A number $R \geq 0$ is an achievable SK rate if for every $\epsilon>0$, there exist local randomization $U_{1}, U_{2}, U_{3}$, communication-transmission protocol $\mathbf{F}$ and $\epsilon$-SK $K$ with

$$
\frac{1}{n} \log |\mathcal{K}| \geq R
$$

for all $n$ sufficiently large.

The supremum of all achievable SK rates is called the SK capacity, denoted by $C$.

The general problem of characterizing $C$ remains open. In [6], general lower bounds and upper bounds for $C$ were given; we state the former next, specialized for the case of two input MAC.

Theorem 1. 6] The SK capacity for a MAC is bounded below as

$$
C \geq R^{*}
$$

For the special case $W\left(x_{3} \mid x_{1}, x_{2}\right)=\mathbb{1}\left(x_{3}=x_{1} \oplus x_{2}\right)$, the lower bound above is tight and $C=R^{*}$ [6, Example 1]. Also, for the case when $W$ is in Willems class of MACs [12], an upper bound for $C$ was derived in [6]. Willems class consists of MAC where one of the inputs, say input 1 , is determined by the output and the other input, i.e., for some mapping $\phi: \mathcal{X}_{2} \times \mathcal{X}_{3} \rightarrow \mathcal{X}_{1}, W\left(x_{3} \mid x_{1}, x_{2}\right)=0$ if $x_{1} \neq$ $\phi\left(x_{2}, x_{3}\right)$. The following result holds.

Theorem 2. [6] For a MAC in Willems class,

$$
C \leq R_{f}^{*}
$$

In this paper, we show that the bounds and (9) are tight under various restrictions imposed on the MAC and the communication-transmission protocols. We first describe the specific restrictions we place. As in Definition 11 define the SK capacity with source emulation [5], [6], [3], denoted by $C_{\mathrm{SE}}$, as the supremum of all achievable SK rates with the additional restriction that

$$
F_{t}=\text { constant }, \quad 2 \leq t \leq n,
$$

i.e., the transmission input sequences for the MAC are selected solely based on the initial interactive communication 
$F_{1}$ and local randomization at the input terminals, without any feedback from the output. Next, define the SK capacity with no input communication, denoted by $C_{\mathrm{NIC}}$, as the supremum of all achievable SK rates with the additional restriction that following the first round interactive communication $F_{1}$, the subsequent communication $F_{2}, \ldots, F_{n}$ are only from the output terminal, i.e.,

$$
F_{t}=F_{t}\left(U_{3}, X_{3}^{t-1}, F^{t-1}\right), \quad 2 \leq t \leq n .
$$

The following inequalities ensue:

$$
C_{\mathrm{SE}} \leq C_{\mathrm{NIC}} \leq C .
$$

We now state our main results. First, we show a general upper bound on $C_{\mathrm{NIC}}$.

Theorem 3. The SK capacity with no input communication is bounded above as

$$
C_{\mathrm{NIC}} \leq R_{f}^{*}
$$

Next, we show that for the class of symmetric MACs, this upper bound is tight.

Theorem 4. For a symmetric MAC with $\mathcal{X}_{1}=\mathcal{X}_{2}$ and

$$
W\left(x_{3} \mid x_{1}, x_{2}\right)=W\left(x_{3} \mid x_{2}, x_{1}\right),
$$

the SK capacity with no input communication is given by

$$
C_{\mathrm{NIC}}=R_{f}^{*} .
$$

As a corollary, we characterize $C$ for adder MAC, for which lower and upper bounds were reported in [6, Example 2].

Corollary. For $W\left(x_{3} \mid x_{1}, x_{2}\right)=\mathbb{1}\left(x_{3}=x_{1}+x_{2}\right)$, the $S K$ capacity is given by

$$
C=R_{f}^{*}
$$

Since adder MAC is in Willems class and is symmetric, the corollary follows from Theorem 2 and Theorem 4

Finally, the following result implies that source emulation does not suffice to generate SKs of rate $R_{f}^{*}$ and the complex communication-transmission protocols above are needed necessarily in Theorem 4

Theorem 5. The SK capacity with source emulation is given by

$$
C_{\mathrm{SE}}=R^{*} .
$$

The inequality $C_{\mathrm{SE}} \geq R^{*}$ was shown in [6]. We show the reverse inequality in Section $\mathrm{V}$

Remark. Theorem 5 is a further strengthening of [6, Proposition 5] where this result was established for source emulation protocols that restrict the inputs of the MAC for different channel uses to be i.i.d. We show that the inequality $C_{\mathrm{SE}} \leq R^{*}$ holds even when this restriction is dropped.

\section{A General Converse for SK CAPACity of A Multiterminal SOURCE}

In this section, we present a converse for an SK generation problem in a multiterminal source model with $m$ sources (c.f.
[4]) that does not require the underlying sources to be i.i.d. This specific form of the converse is due to Prakash Narayan and it relies on a basic property of interactive communication in multiterminal models shown in [5].

Terminals $1, \ldots, m$ observe correlated rvs $Y_{1}, \ldots, Y_{m}$, respectively; for brevity we denote by $\mathcal{M}$ the set $\{1, \ldots, m\}$ and by $Y_{A}$ the rvs $\left\{Y_{i}, i \in A\right\}$ for $A \subseteq \mathcal{M}$. The terminals communicate over a public channel, possibly interactively in several rounds. Specifically, terminal $i$ sends communication $F_{i j}$ in the $j$ th round, $1 \leq j \leq r$, where $F_{i j}$ depends on the observation $Y_{i}$ and the previously received communication

$$
F_{11}, \ldots, F_{m 1}, \ldots, F_{1 j}, \ldots, F_{(i-1) j} .
$$

We denote the overall interactive communication by $\mathbf{F}$. Consider an rv $K$ taking values in $\mathcal{K}$ such that

$$
\mathrm{P}\left(K=K_{i}\left(Y_{i}, \mathbf{F}\right), i \in \mathcal{M}\right) \geq 1-\epsilon,
$$

for $0<\epsilon<1$ and some mappings $K_{i}$ of $\left(Y_{i}, \mathbf{F}\right)$, i.e., the terminals form estimates of $K$ using their respective observations $Y_{i}$ and the interactive communication $\mathbf{F}$ that agree with $K$ with probability greater than $1-\epsilon$. We present below an upper bound on $\log |\mathcal{K}|$. The following notations will be used: Let $\mathcal{B}$ be a collection of subsets of $\mathcal{M}$ given by

$$
\mathcal{B}=\{B: B \subsetneq \mathcal{M}, B \neq \emptyset\} .
$$

A collection $\lambda=\left\{\lambda_{B} \in[0,1]: B \in \mathcal{B}\right\}$ constitutes a fractional partition of $\mathcal{M}$ (c.f. [5]) if

$$
\sum_{B \in \mathcal{B}: i \in B} \lambda_{B}=1, \quad \text { for all } i \in \mathcal{M} .
$$

Consider a partition $\pi=\left\{\pi_{1}, \ldots, \pi_{k}\right\}$ of $\mathcal{M}$. Corresponding to this partition, we define a fractional partition $\lambda^{\pi}$ as follows:

$$
\lambda_{B}^{\pi}= \begin{cases}\frac{1}{k-1}, & B=\pi_{i}^{c}, 1 \leq i \leq k, \\ 0, & \text { otherwise. }\end{cases}
$$

First, we present a key property of interactive communication that underlies all the converse proofs of this paper.

Lemma 6 (Interactive Communication Property). [5] For an interactive communication $\mathbf{F}$, we have

$$
H(\mathbf{F}) \geq \sum_{B \in \mathcal{B}} \lambda_{B} H\left(\mathbf{F} \mid Y_{B^{c}}\right),
$$

for every fractional partition $\lambda$ of $\mathcal{M}$.

The following result is, in effect, a "single-shot" converse for the SK generation problem.

Theorem 7. 9] For an $r v K$ and interactive communication F satisfying (10), we have

$\log |\mathcal{K}| \leq H\left(Y_{\mathcal{M}}\right)-\sum_{B \in \mathcal{B}} \lambda_{B} H\left(Y_{B} \mid Y_{B^{c}}\right)+s_{i n}(K ; \mathbf{F})+\nu$,

for every fractional partition $\lambda$ of $\mathcal{M}$, where $\nu=(m+$ $2)(\epsilon \log |\mathcal{K}|+h(\epsilon))$. 
Proof. It follows from [5, Lemma A.2] that

$$
\begin{aligned}
H(K \mid \mathbf{F}) \leq & H\left(Y_{\mathcal{M}} \mid \mathbf{F}\right)-\sum_{B \in \mathcal{B}} \lambda_{B} H\left(Y_{B} \mid Y_{B^{c}}, \mathbf{F}\right)+\nu, \\
= & H\left(Y_{\mathcal{M}}\right)-\sum_{B \in \mathcal{B}} \lambda_{B} H\left(Y_{B} \mid Y_{B^{c}}\right) \\
& -\left[H(\mathbf{F})-\sum_{B \in \mathcal{B}} \lambda_{B} H\left(\mathbf{F} \mid Y_{B^{c}}\right)\right]+\nu,
\end{aligned}
$$

which, along with Lemma 6 and the definition of $s_{i n}(K ; \mathbf{F})$ in (6), completes the proof.

Corollary. For $K$ and $\mathbf{F}$ as in Theorem 7 we get

$$
\log |\mathcal{K}| \leq \frac{1}{k-1} D\left(\mathrm{P}_{Y_{\mathcal{M}}} \| \prod_{i=1}^{k} \mathrm{P}_{Y_{\pi_{i}}}\right)+s_{i n}(K ; \mathbf{F})+\nu
$$

for every partition $\pi=\left\{\pi_{1}, \ldots, \pi_{k}\right\}$ of $\mathcal{M}$.

The corollary follows upon choosing $\lambda=\lambda^{\pi}$ in Theorem 7 where $\lambda^{\pi}$ is given by 11 .

\section{MAXimum Symmetric RATE FOR MAC}

While a single-letter expression for $R^{*}$ is known [1], [7], for $R_{f}^{*}$ such an expression is available only in special cases [12]. In this section, we will present $n$-letter characterizations for $R^{*}$ and $R_{f}^{*}$, which will be used in our proofs in the next section.

Lemma 8. For MAC with two inputs,

$$
\begin{aligned}
R^{*}=\varlimsup_{n} \sup \min \{ & \frac{1}{n} I\left(X_{1}^{n} \wedge X_{3}^{n} \mid X_{2}^{n}\right), \\
& \frac{1}{n} I\left(X_{2}^{n} \wedge X_{3}^{n} \mid X_{1}^{n}\right), \\
& \left.\frac{1}{2 n} I\left(X_{1}^{n}, X_{2}^{n} \wedge X_{3}^{n}\right)\right\},
\end{aligned}
$$

where the sup is over all distributions $\mathrm{P}_{X_{1}^{n} X_{2}^{n} X_{3}^{n}}=$ $\mathrm{P}_{X_{1}^{n}} \mathrm{P}_{X_{2}^{n}} W^{n}$.

We omit the proof, which is a simple consequence of the capacity region for a MAC [1], [7].

Lemma 9. For MAC with two inputs,

$$
\begin{aligned}
R_{f}^{*}=\varlimsup_{n} \sup \min \{ & \frac{1}{n} I\left(U_{1} \wedge X_{3}^{n}, U_{3} \mid U_{2}\right), \\
& \frac{1}{n} I\left(U_{2} \wedge X_{3}^{n}, U_{3} \mid U_{1}\right), \\
& \left.\frac{1}{2 n} I\left(U_{1}, U_{2} \wedge X_{3}^{n}, U_{3}\right)\right\},
\end{aligned}
$$

where the sup is over all joint distributions $U_{1}, U_{2}, U_{3}, X_{3}^{n}$ of the randomization at the terminals and the output of the $M A C$ that result from communication-transmission protocols with no input communication (as in the definition of $C_{\mathrm{NIC}}$ ).

Proof. First, we claim that making additional independent common randomness $U_{3}$ available to the senders and the receiver does not improve the capacity region of a MAC. Indeed, let $\mathrm{P}_{\text {err }}\left(u_{3}\right)$ be the error probability of the MAC $W^{n}$ with feedback conditioned on $U_{3}=u_{3}$. Clearly, there exists at least one realization $u_{3}^{*}$ such that

$$
\mathrm{P}_{\mathrm{err}}\left(u_{3}^{*}\right) \leq \mathbb{E}\left[\mathrm{P}_{\mathrm{err}}\left(U_{3}\right)\right]
$$

Thus, using the encoders and decoders with $U_{3}=u_{3}^{*}$ fixed we can achieve the same rate as that of the original scheme. In the remainder of the proof, without loss of generality, we will assume the availability of rv $U_{3}$ to the senders and the receiver of the MAC.

If $(R, R) \in \mathcal{C}_{\mathrm{MACFB}}$, then using standard manipulations and Fano's inequality we get

$$
R \leq \frac{1}{n} I\left(U_{1} \wedge X_{3}^{n}, U_{3} \mid U_{2}\right)+\eta_{n},
$$

where $U_{1}, U_{2}$ are the messages sent by terminal 1 and 2 , respectively, i.i.d. uniform over $\left\{1, \ldots,\left\lfloor 2^{n} R\right\rfloor\right\}$, and $\eta_{n} \rightarrow 0$ as $n \rightarrow \infty$. Also,

$$
R \leq \frac{1}{n} I\left(U_{2} \wedge X_{3}^{n}, U_{3} \mid U_{1}\right)+\eta_{n},
$$

and

$$
2 R \leq \frac{1}{n} I\left(U_{1}, U_{2} \wedge X_{3}^{n}, U_{3}\right)+\eta_{n} .
$$

Since a code for MAC with feedback constitutes a valid communication-transmission protocol with local randomization $U_{1}, U_{2}, U_{3}$ at terminals $1,2,3$, respectively, it follows that $R_{f}^{*}$ is bounded above by the right-side of 12 .

For the other direction, consider a $\operatorname{MAC} W^{(n)}: \mathcal{U}_{1} \times \mathcal{U}_{2} \rightarrow$ $\mathcal{X}_{3}^{n} \times \mathcal{U}_{3}$ given by

$$
\begin{aligned}
& W^{(n)}\left(x_{3}^{n}, u_{3} \mid u_{1}, u_{2}\right) \\
& =\mathrm{P}\left(X_{3}^{n}=x_{3}^{n}, U_{3}=u_{3} \mid U_{1}=u_{1}, U_{2}=u_{2}\right) .
\end{aligned}
$$

Then, by [1] and [7], the right-side of (12) is less than the maximum symmetric rate of the messages that can be transmitted reliably over this MAC (without feedback). To complete the proof we note that we can simulate $W^{(n)}$ by using the MAC $W$ with feedback $n$ times. Specifically, given a communication-transmission protocol with no input communication and fixed values $u_{1}, u_{2}, u_{3}$, choosing

$$
\begin{aligned}
& X_{1 t}=X_{1 t}\left(u_{1}, F^{t-1}\left(x_{3}^{t-1}, u_{3}\right)\right), \\
& X_{2 t}=X_{2 t}\left(u_{2}, F^{t-1}\left(x_{3}^{t-1}, u_{3}\right)\right), \quad 1 \leq t \leq n .
\end{aligned}
$$

simulates $W^{(n)}$. This is a valid choice of inputs since both the senders know the common randomness $U_{3}$ and the feedback signals $X_{3}^{t-1}$ at time $t$.

\section{UPPER BOUNDS}

In this section, we prove upper bounds on $C_{\mathrm{NIC}}$ and $C_{\mathrm{SE}}$ by applying the results developed in Sections [I and IV] We assume that the SK satisfies the "weak secrecy" condition (7).

The following observation from [11] is needed.

Lemma 10. For mutually independent rvs $Y_{1}, Y_{2}, Y_{3}$ and an interactive communication $\mathbf{F}$ for the sources $Y_{1}, Y_{2}, Y_{3}$ 
described in Section III we have

$$
\mathrm{P}_{Y_{1}, Y_{2}, Y_{3} \mid \mathbf{F}}\left(y_{1}, y_{2}, y_{3} \mid \mathbf{f}\right)=\prod_{i=1}^{3} \mathrm{P}_{Y_{i} \mid \mathbf{F}}\left(y_{i} \mid \mathbf{f}\right), \quad \forall \mathbf{f},
$$

i.e., independent observations remain independent when conditioned on an interactive communication.

We first remark that the initial round of interactive communication $F_{1}$ does not help. Specifically, for an $\epsilon$-SK $K$ recoverable from an interactive communication $\mathbf{F}$, it follows from (5) and (6) that there exists a fixed value $f_{1}$ of $F_{1}$ such that

$$
\begin{gathered}
\mathrm{P}\left(K_{1}=K_{2}=K_{3}=K \mid F_{1}=f_{1}\right) \geq 1-2 \epsilon, \\
\log |\mathcal{K}|-H\left(K \mid \mathbf{F}, F_{1}=f_{1}\right) \leq 2 \epsilon
\end{gathered}
$$

Note that by Lemma 10 the rvs $U_{1}, U_{2}, U_{3}$ are conditionally independent given $F_{1}$. Consider a modified protocol obtained by fixing $F_{1}=f_{1}$ and using local randomization $\tilde{U}_{1}, \tilde{U}_{2}, \tilde{U}_{3}$ with the same distribution as the conditional distribution of $U_{1}, U_{2}, U_{3}$ given $F_{1}=f_{1}$. Then, in view of (13), the modified protocol generates a $2 \epsilon-\mathrm{SK}$ of rate not less than the original protocol and does not require any initial interactive communication. Thus, without loss of generality, in the remainder of the section we assume that $F_{1}$ is constant.

\section{A. Proof of $C_{\mathrm{NIC}} \leq R_{f}^{*}$}

Let $R$ be an achievable SK rate for a MAC with no input communication. Setting $Y_{1}=U_{1}, Y_{2}=U_{2}$ and $Y_{3}=\left(X_{3}^{n}, U_{3}\right)$ and applying the corollary to Theorem 7 with partition $\pi=(\{1\},\{2,3\})$, for every $\delta>0$ and $n$ sufficiently large we have

$$
\begin{aligned}
R & \leq \frac{1}{n} D\left(\mathrm{P}_{U_{1} U_{2} X_{3}^{n} U_{3}} \| \mathrm{P}_{U_{1}} \times \mathrm{P}_{U_{2} X_{3}^{n} U_{3}}\right)+\delta \\
& =\frac{1}{n} I\left(U_{1} \wedge U_{2}, X_{3}^{n}, U_{3}\right)+\delta \\
& =\frac{1}{n} I\left(U_{1} \wedge X_{3}^{n}, U_{3} \mid U_{2}\right)+\delta,
\end{aligned}
$$

and similarly, using the partition $\pi=(\{2\},\{1,3\})$,

$$
R \leq \frac{1}{n} I\left(U_{2} \wedge X_{3}^{n}, U_{3} \mid U_{1}\right)+\delta .
$$

Also, for the partition $\pi=(\{1\},\{2\},\{3\})$, we get for $n$ large

$$
\begin{aligned}
R & \leq \frac{1}{2 n} D\left(\mathrm{P}_{U_{1} U_{2} X_{3}^{n} U_{3}} \| \mathrm{P}_{U_{1}} \times \mathrm{P}_{U_{2}} \times \mathrm{P}_{X_{3}^{n} U_{3}}\right)+\delta \\
& =\frac{1}{2 n} I\left(U_{1}, U_{2} \wedge X_{3}^{n}, U_{3}\right)+\delta,
\end{aligned}
$$

where the equality uses the independence of $U_{1}$ and $U_{2}$. Upon combining the bounds in 14 - 16 and taking the limit $n \rightarrow \infty$, an application of Lemma 9 yields

$$
R \leq R_{f}^{*}
$$

since $\delta>0$ was arbitrary. This proves the claimed upper bound.
Remark. Choosing $\pi=(\{1,2\},\{3\})$, we also get the bound

$$
\begin{aligned}
R & \leq \frac{1}{n} D\left(\mathrm{P}_{U_{1} U_{2} X_{3}^{n} U_{3}} \| \mathrm{P}_{U_{1} U_{2}} \times \mathrm{P}_{X_{3}^{n} U_{3}}\right)+\delta \\
& =\frac{1}{n} I\left(U_{1}, U_{2} \wedge X_{3}^{n}, U_{3}\right)+\delta
\end{aligned}
$$

which is subsumed by 16 .

\section{B. Proof of $C_{\mathrm{SE}} \leq R^{*}$}

Let $R$ be an achievable SK rate for a MAC with source emulation. Setting $Y_{1}=\left(X_{1}^{n}, U_{1}\right), Y_{2}=\left(X_{2}^{n}, U_{2}\right)$ and $Y_{3}=$ $\left(X_{3}^{n}, U_{3}\right)$, and following the steps of the previous part mutatis mutandis, we get

$$
\begin{aligned}
R \leq \varlimsup_{n} \sup \min \{ & \frac{1}{n} I\left(X_{1}^{n}, U_{1} \wedge X_{3}^{n}, U_{3} \mid X_{2}^{n}, U_{2}\right), \\
& \frac{1}{n} I\left(X_{2}^{n}, U_{2} \wedge X_{3}^{n}, U_{3} \mid X_{1}^{n}, U_{1}\right), \\
& \left.\frac{1}{2 n} I\left(X_{1}^{n}, U_{1}, X_{2}^{n}, U_{2} \wedge X_{3}^{n}, U_{3}\right)\right\} .
\end{aligned}
$$

Note that

$$
\begin{aligned}
& I\left(X_{1}^{n}, U_{1} \wedge X_{3}^{n}, U_{3} \mid X_{2}^{n}, U_{2}\right) \\
& =I\left(X_{1}^{n}, U_{1} \wedge X_{3}^{n} \mid X_{2}^{n}, U_{2}\right) \\
& \leq I\left(X_{1}^{n} \wedge X_{3}^{n} \mid X_{2}^{n}\right),
\end{aligned}
$$

where the equality follows since $U_{3}$ is independent of the rest of the rvs, and the inequality 5 uses $U_{1}, U_{2} \bullet X_{1}^{n}, X_{2}^{n} \bullet X_{3}^{n}$. Similarly,

$$
I\left(X_{2}^{n}, U_{2} \wedge X_{3}^{n}, U_{3} \mid X_{1}^{n}, U_{1}\right) \leq I\left(X_{2}^{n} \wedge X_{3}^{n} \mid X_{1}^{n}\right),
$$

and

$$
I\left(X_{1}, U_{1}, X_{2}^{n}, U_{2} \wedge X_{3}^{n}, U_{3}\right) \leq I\left(X_{1}^{n}, X_{2}^{n} \wedge X_{3}^{n}\right),
$$

where the rvs $X_{1}^{n}=X_{1}^{n}\left(U_{1}\right)$ and $X_{2}^{n}=X_{2}^{n}\left(U_{2}\right)$ are independent. By Lemma 8 and $(17)$, the upper bound on $C_{\mathrm{SE}}$ follows.

\section{LOWER BOUNDS}

In this section, we prove Theorem 4 Suppose $(R, R)$ lies in $\mathcal{C}_{\text {MACFB }}$ for a symmetric MAC. Then, there exist encoder mappings

$$
\begin{aligned}
& \tau_{1 t}:\left\{1, \ldots,\left\lfloor 2^{n R}\right\rfloor\right\} \times \mathcal{X}_{3}^{t-1} \rightarrow \mathcal{X}_{1}, \\
& \tau_{2 t}:\left\{1, \ldots,\left\lfloor 2^{n R}\right\rfloor\right\} \times \mathcal{X}_{3}^{t-1} \rightarrow \mathcal{X}_{2}, \quad 1 \leq t \leq n,
\end{aligned}
$$

and decoder mapping

$$
\rho: \mathcal{X}_{3}^{n} \rightarrow\left\{1, \ldots,\left\lfloor 2^{n R}\right\rfloor\right\} \times\left\{1, \ldots,\left\lfloor 2^{n R}\right\rfloor\right\}
$$

such that when messages $M_{1}, M_{2}$ are sent, where rvs $M_{1}$ and $M_{2}$ are i.i.d. uniform over $\left\{1, \ldots,\left\lfloor 2^{n R}\right\rfloor\right\}$, the error probability satisfies

$$
\epsilon_{n}=\mathrm{P}\left(\rho\left(X_{3}^{n}\right) \neq\left(M_{1}, M_{2}\right)\right) \rightarrow 0,
$$

in the limit as $n \rightarrow \infty$.

Using this $n$ length code, we construct a symmetric code of length $2 n$ by applying (19) and 20 twice as

\footnotetext{
${ }^{5}$ In fact, the inequality holds with equality.
} 
follows. Consider rvs $\hat{M}_{1}, \hat{M}_{2}, \tilde{M}_{1}, \tilde{M}_{2}$ i.i.d. uniform over $\left\{1, \ldots,\left\lfloor 2^{n R}\right\rfloor\right\}$. We send inputs corresponding to messages $\hat{M}_{1}, \hat{M}_{2}$ in the odd time instances, and, with the roles of $\tau_{1 t}$ and $\tau_{2 t}$ interchanged, send inputs corresponding to messages $\tilde{M}_{1}, \tilde{M}_{2}$ in the even time instances. Using the outputs at the odd and even time instances to decode $\hat{M}_{1}, \hat{M}_{2}$ and $\tilde{M}_{1}$, $\tilde{M}_{2}$, respectively, we obtain a code of rate $(R, R)$ with error probability bounded above by $2 \epsilon_{n}$. Denoting by $Y_{t}$ the rv $\left(X_{3(2 t-1)}, X_{3(2 t)}\right), 1 \leq t \leq n$, and letting $M_{1}=\left(\hat{M}_{1}, \tilde{M}_{1}\right)$ and $M_{2}=\left(\hat{M}_{2}, \tilde{M}_{2}\right)$, we get

$$
\begin{aligned}
H\left(Y_{t} \mid M_{1}, Y^{t-1}\right) & \\
=H & \left(X_{3(2 t-1)} \mid \hat{M}_{1}, X_{31}, \ldots, X_{3(2 t-3)}\right) \\
& \quad+H\left(X_{3(2 t)} \mid \tilde{M}_{1}, X_{32}, \ldots, X_{3(2 t-2)}\right) \\
=H & \left(X_{3(2 t)} \mid \tilde{M}_{2}, X_{32}, \ldots, X_{3(2 t-2)}\right) \\
& \quad+H\left(X_{3(2 t-1)} \mid \hat{M}_{2}, X_{31}, \ldots, X_{3(2 t-3)}\right) \\
=H & \left(Y_{t} \mid M_{2}, Y^{t-1}\right),
\end{aligned}
$$

where the second equality follows from the symmetry of the MAC.

Next, we replace the feedback $Y_{t}$ with its compressed version given the observations of the input terminals. To do this, we consider a multiple-blocks extension of the symmetric code above and take recourse to the result of Slepian and Wolf [10]. Specifically, let $M_{1 i}, M_{2 i}, Y_{i}^{n}, i=1, \ldots, N$, be $N$ i.i.d. repetitions of rvs $M_{1}, M_{2}, Y^{n}$ above. By Slepian-Wolf theorem [10], there exist mappings

$$
F_{t}=F_{t}\left(Y_{t 1}, Y_{t 2}, \ldots, Y_{t N}\right), \quad 1 \leq t \leq n,
$$

of rates

$$
\begin{aligned}
\frac{1}{N} \log \left\|F_{t}\right\| & \leq H\left(Y_{t} \mid M_{1}, Y^{t-1}\right)+\epsilon_{n}, \\
& =H\left(Y_{t} \mid M_{2}, Y^{t-1}\right)+\epsilon_{n},
\end{aligned}
$$

such that an observer of $\left(M_{11}, \ldots, M_{1 N}, Y_{1}^{t-1}, \ldots, Y_{N}^{t-1}\right)$ or $\left(M_{21}, \ldots, M_{2 N}, Y_{1}^{t-1}, \ldots, Y_{N}^{t-1}\right)$ can recover $Y_{t}^{N}$ with probability of error less than $\epsilon_{n} / n$, for all $N$ sufficiently large. The equality in (22) uses (21). Then, using a union bound on probability of error, the communication-transmission protocol corresponding to $F_{1}, \ldots, F_{n}$ allows all the terminals to recover $\left(Y_{1}^{n}, \ldots, Y_{N}^{n}\right)$ with probability of error less than $\epsilon_{n}$. Note that the overall communication-transmission protocol now consists of $n$ rounds of communication from terminal 3 and $2 n N$ transmissions over the MAC. In each time slot $t$, the output terminal observing $Y_{t 1}, \ldots, Y_{t N}$ sends $F_{t}$ to the input terminals. Using this communication and their local observations $M_{1}^{N}$ and $M_{2}^{N}$, the terminals 1 and 2 estimate $Y_{t 1}, \ldots, Y_{t N}$ and use the estimates to select the inputs $X_{1(2 t+1)}^{N}, X_{1(2 t+2)}^{N}$ and $X_{2(2 t+1)}^{N}, X_{2(2 t+2)}^{N}$, respectively.

Finally, we show that for all $n, N$ sufficiently large, there exists a function $K$ of $\left(Y_{1}^{n}, \ldots, Y_{N}^{n}\right)$ of rate $(1 / n N) \log \|K\|$ greater than $R-\delta$, satisfying

$$
s_{i n}(K ; \mathbf{F}) \leq \epsilon .
$$

Therefore, $K$ is an $\epsilon$-SK for $n, N$ sufficiently large, where $0<\epsilon<1$ is arbitrary. It remains to find a mapping $K$ as above. By [4, Lemma 1], it suffices to show that

$$
\left\|\mathrm{P}_{K \mathbf{F}}-\mathrm{P}_{\text {unif }} \times \mathrm{P}_{\mathbf{F}}\right\| \leq 2^{-n \tau},
$$

for some $\tau>0$. Indeed, by the "balanced coloring lemma" [4. Lemma B4], for $n, N$ sufficiently large, there exists such a mapping $K$ of rate

$$
\begin{aligned}
\frac{1}{n N} \log \|K\| & \geq \frac{1}{n N} H\left(Y_{1}^{n}, \ldots, Y_{N}^{n}\right)-\frac{1}{n N} \log \|\mathbf{F}\|-\epsilon_{n} \\
& \geq \frac{1}{n} H\left(Y^{n}\right)-\frac{1}{n} \sum_{t=1}^{n} H\left(Y_{t} \mid M_{1}, Y^{t-1}\right)-2 \epsilon_{n} \\
& =\frac{1}{n} I\left(Y^{n} \wedge M_{1}\right)-2 \epsilon_{n} \\
& \geq R-\delta,
\end{aligned}
$$

where the second inequality is by (22) and the previous inequality uses Fano's inequality. Thus, $R$ is an achievable SK rate.

\section{DISCUSSION}

Our proof methodology in this paper is to use the basic properties of SKs and interactive communication to obtain upper bounds on SK rates, and then relate these upper bounds directly to the maximum rates of reliable transmission over a MAC, without reducing them to single-letter forms. In particular, this approach brings out a key property of interactive communication that is instrumental in proving the converse, namely the inequality (see Lemma 6)

$$
H(\mathbf{F}) \geq \sum_{B \in \mathcal{B}} \lambda_{B} H\left(\mathbf{F} \mid Y_{B^{c}}\right) .
$$

For the case of two terminals, this inequality can be written as

$$
H(\mathbf{F}) \geq H\left(\mathbf{F} \mid Y_{1}\right)+H\left(\mathbf{F} \mid Y_{2}\right),
$$

which is well-known in the communication complexity literature (c.f. [2]) as the fact that external information cost is at least as much as the information cost. Besides (23), the only other property of interactive communication that we use is the fact that independent observations remain so when conditioned on interactive communication (see Lemma 10). However, for a specific choice of $\lambda$ in (23), upon rearranging the terms we get

$$
I\left(Y_{B} \wedge Y_{B^{c}} \mid \mathbf{F}\right) \leq I\left(Y_{B} \wedge Y_{B^{c}}\right), \quad \text { for all } B \subseteq \mathcal{M},
$$

which in turn implies Lemma 10. Thus, 231) is the only property of interactive communication that is used in our converse proofs. Note that (24) is indeed a characteristic of an interactive communication and does not hold for every function of $Y_{1}$ and $Y_{2}$. For instance, for symmetrically distributed unbiased bits $Y_{1}$ and $Y_{2}$, and $F=Y_{1} \oplus Y_{2}$,

$$
H(F)=1<H\left(F \mid Y_{1}\right)+H\left(F \mid Y_{2}\right)=2 .
$$

Our results in this paper extend easily to MACs with multiple inputs. In particular, Theorems 3 and 5 hold for 
a multi-input MAC upon defining $R^{*}$ and $R_{f}^{*}$ as follows:

$$
\begin{aligned}
& R^{*}=\max \left\{R:(R, \ldots, R) \in \mathcal{C}_{\mathrm{MAC}}\right\}, \\
& R_{f}^{*}=\max \left\{R:(R, \ldots, R) \in \mathcal{C}_{\mathrm{MACFB}}\right\} .
\end{aligned}
$$

Also, Theorem 4 holds for a multi-input MAC $W: \mathcal{X}_{1} \times$ $\ldots \times \mathcal{X}_{m-1} \rightarrow \mathcal{X}_{m}$ that satisfies

$$
W\left(x_{m} \mid x_{1}, \ldots, x_{m-1}\right)=W\left(x_{m} \mid x_{\sigma(1)}, \ldots, x_{\sigma(m-1)}\right),
$$

for every permutation $\sigma$ of $\{1, \ldots, m-1\}$.

\section{ACKNOWLEDGEMENTS}

The authors are indebted to Prakash Narayan for allowing us to include Theorem 7 in this paper. Also, the discussion on interactive communication properties in the last section is based on ideas developed jointly with him.

\section{REFERENCES}

[1] R. Ahlswede, "Multiway communication channels," Proc. IEEE International Symposium on Information Theory, pp. 23-52, 1973.

[2] M. Braverman, "Coding for interactive computation: progress and challenges," Proc. Conference on Communication, Control, and Computing (Allerton), pp. 1914-1921, October 2012.
[3] C. Chan, "Generating secret in a network," Ph. D. Dissertation, Massachussetts Institute of Technology, 2010.

[4] I. Csiszár and P. Narayan, "Secrecy capacities for multiple terminals," IEEE Trans. Inf. Theory, vol. 50, no. 12, pp. 3047-3061, December 2004.

[5] — "Secrecy capacities for multiterminal channel models," IEEE Trans. Inf. Theory, vol. 54, no. 6, pp. 2437-2452, June 2008.

[6] - "Secrecy generation for multiaccess channel models," IEEE Trans. Inf. Theory, vol. 59, no. 1, pp. 17-31, 2013.

[7] H. Liao, "A coding theorem for multipleaccess communications," Proc. IEEE International Symposium on Information Theory, 1972.

[8] M. Madiman and P. Tetali, "Information inequalities for joint distributions, with interpretations and applications," IEEE Trans. Inf. Theory, vol. 56, no. 6, pp. 2699-2713, June 2010.

[9] P. Narayan, Personal Communication, 2013.

[10] D. Slepian and J. Wolf, "Noiseless coding of correlated information source," IEEE Trans. Inf. Theory, vol. 19, no. 4, pp. 471-480, July 1973.

[11] H. Tyagi and P. Narayan, "How many queries will resolve common randomness?" To appear, IEEE Trans. Inf. Theory, 2013.

[12] F. M. J. Willems, "The feedback capacity region of a class of discrete memoryless multiple access channels," IEEE Trans. Inf. Theory, vol. 28, no. 1, pp. 93-95, January 1982. 